\title{
Ganoderma lucidum polysaccharides protect fibroblasts against UVB-induced photoaging
}

\author{
QINGHAI ZENG $^{1 *}$, FANG ZHOU $^{1 *}$, LI LEI $^{1-3^{*}}$, JING CHEN $^{1}$, JIANYUN LU $^{1}$, \\ JIANDA ZHOU ${ }^{4}, \mathrm{KE} \mathrm{CAO}^{5}$, LIHUA GAO ${ }^{1}$, FANG XIA $^{5}$, SHU DING $^{1}$, LIHUA HUANG $^{6}$, \\ HONG XIANG $^{6}$, JINGJING WANG ${ }^{1}$, YANGFAN XIAO ${ }^{7}$, RONG XIAO ${ }^{7}$ and JINHUA HUANG ${ }^{1}$ \\ ${ }^{1}$ Department of Dermatology, Third Xiangya Hospital; \\ ${ }^{2}$ Department of Dermatology and ${ }^{3}$ Hunan Key Laboratory of Skin Cancer and Psoriasis, Xiangya Hospital; \\ Departments of ${ }^{4}$ Burn and Plastic Surgery, ${ }^{5}$ Oncology, and ${ }^{6}$ The Central Laboratory, Third Xiangya Hospital; \\ ${ }^{7}$ Department of Dermatology, Second Xiangya Hospital, Central South University, Changsha, Hunan 410013, P.R. China
}

Received October 22, 2015; Accepted October 28, 2016

DOI: $10.3892 / \mathrm{mmr} .2016 .6026$

\begin{abstract}
Ganoderma lucidum has featured in traditional Chinese medicine for $>1,000$ years. Ganoderma polysaccharides (GL-PS), a major active ingredient in Ganoderma, confer immune regulation, antitumor effects and significant antioxidant effects. The aim of the present study was to investigate the efficacy and mechanism of GL-PS-associated inhibition of ultraviolet B (UVB)-induced photoaging in human fibroblasts in vitro. Primary human skin fibroblasts were cultured, and a fibroblast photoaging model was built through exposure to UVB. Cell viability was measured by MTT assay. Aged cells were stained using a senescence-associated $\beta$-galactosidase staining (SA- $\beta$-gal) kit. ELISA kits were used to analyze matrix metalloproteinase (MMP) -1 and C-telopeptides of Type I collagen (CICP) protein levels in cellular supernatant ROS levels were quantified by flow cytometry. Cells exposed to UVB had decreased cell viability, increased aged cells, decreased CICP protein expression, increased MMP-1 protein expression, and increased cellular ROS levels compared with non-exposed cells. However, cells exposed to UVB and treated with 10,20 and $40 \mu \mathrm{g} / \mathrm{ml}$ GL-PS demonstrated increased cell viability, decreased aged cells, increased CICP protein expression, decreased MMP-1 protein expression, and decreased cellular ROS levels compared with UVB exposed/GL-PS untreated cells. These results demonstrate that GL-PS protects fibroblasts against photoaging by eliminating UVB-induced
\end{abstract}

Correspondence to: Professor Jinhua Huang, Department of Dermatology, Third Xiangya Hospital, Central South University, 138 Tongzipo Road, Changsha, Hunan 410013, P.R. China

E-mail: huangjinhua60@163.com

*Contributed equally

Key words: Ganoderma lucidum polysaccharides, fibroblast, photoaging, ROS
ROS. This finding indicates GL-PS treatment may serve as a novel strategy for antiphotoaging.

\section{Introduction}

Skin photoaging refers to the aging process resulting from exposure to ultraviolet (UV) light (1), characterized by roughening and thickening of the skin, increased flaccidity, coarse wrinkles, local pigmentation, or telangiectasia (2-4). It may also lead to benign or malignant tumors, including sunlight keratosis, squamous cell carcinoma and malignant melanoma (5).

Photoaging has a complex mechanism. Altered levels of reactive oxygen species (ROS), extracellular matrix components, matrix metalloproteinases and cytokines are linked to photoaging, particularly increased ROS levels (6). Upon receiving UV energy, intracellular chromophores interact with oxygen molecules, creating ROS that cause direct cell damage when levels increase above a certain threshold (7-9). Elevated ROS levels also activate a series of signaling pathways through expression of signaling molecules including extracellular signal-regulated kinases (ERKs), c-Jun N-terminal kinase (JNK), and p38 in the mitogen-activated protein kinase (MAPK) pathway. This results in increased matrix metalloproteinase (MMP) expression, and a reduction in collagen protein synthesis and secretion $(10,11)$. Type I collagens are an important component of the extracellular matrix that are degraded by MMP-1 (12). During the photoaging process MMP-1 is overexpressed, resulting in collagen degradation and disintegration (12-16).

Physically covering skin is known to minimize UV damage, but antioxidant and antiinflammatory agents are also increasingly being used $(17,18)$. Current clinical treatments to prevent and delay photoaging rely on photo-dynamic therapy (19) and antioxidants including polyphenols and $\beta$-carotene (20). Previous studies have demonstrated the positive effects of herbal extracts in preventing skin photoaging: Sun et al (21) revealed that wild chrysanthemum extract downregulates ROS levels within HaCat cells and so reduces MMP-2 and MMP-9 expression by inhibiting UV-induced ERK1/2 and p38 
phosphorylation to prevent UVB-related acute skin damage and photoaging. Lee et al (22) demonstrated that macelignan, an effective antioxidant found in the nutmeg, Myristica fragans, downregulates MMP-1 expression by reducing UV exposure-related ROS elevation and MAPK phosphorylation. In addition, it increases type I collagen expression by activating transforming growth factor $\beta$ (TGF- $\beta$ ) /SMAD signaling to effectively prevent and treat skin photoaging.

The fungus Ganoderma lucidum is a famous herbal medicine in China, having featured in traditional Chinese medicine for $>1,000$ years (23-25). G. lucidum polysaccharides (GL-PS) display regulatory abilities toward the immune system, resulting in antitumor and antioxidation effects (26-28). GL-PS has been demonstrated to protect murine skeletal muscles from oxidative stress following exhaustive exercise (29). GL-PS also regulates gene expression in aging skin cells and induces cytokine secretion, suggesting potential anti-aging effects (29). Considering the close relation of photoaging to unbalanced oxidative stress and the antioxidative effect of GL-PS, the present study hypothesized that GL-PS confers protection from photoaging on skin cells.

The present study, to the best of our knowledge, is the first to demonstrate that GL-PS protects fibroblasts from UVB-induced photoaging. The mechanism underlying this may be the reduction of UVB-induced ROS levels, inhibiting the MAPK signal pathway and therefore MMP-1 expression, while promoting type I collagen expression. This suggests that GL-PS may have clinical potential in treating photoaging.

\section{Materials and methods}

Materials. Dulbecco's modified Eagle's medium (DMEM) was obtained from Gibco; Thermo Fisher Scientific, Inc. (Waltham, MA, USA), fetal bovine serum (FBS) from HyClone; GE Healthcare Life Sciences (Logan, UT, USA), 98\% purity GL-PS from Shaanxi Ciyuan Biotech Co., Ltd. (Xi'an, China), senescence-associated $\beta$-galactosidase staining (SA- $\beta$-gal) kit from Beyotime Institute of Biotechnology (Haimen, China), ROS detection kit from Nanjing KeyGen Biotech Co., Ltd. (Nanjing, China), MMP-1 ELISA detection kit (cat. no. EK0458) from Boster Systems, Inc. (Wuhan, China), C-telopeptides of type I collagen (CICP) ELISA detection kit (cat. no. XY-67851Hu) from Shanghai Xin Yu Biotech Co., Ltd (Shanghai, China) and UVB radiation apparatus (SS-07) from Shanghai SIGMA High Tech Co., Ltd. (Shanghai, China).

Fibroblast isolation and culture. Samples were obtained from the donated foreskin of a healthy man (age, 18 years) following circumcision, with informed consent. The foreskin was soaked in iodine for $15 \mathrm{~min}$ and subsequently rinsed with PBS. Subcutaneous tissues were removed, and the remaining tissue was cut into small pieces $(<5 \mathrm{~mm})$. Trypsin was applied to isolate fibroblasts, and all cells were subsequently collected, washed and cultured in DMEM containing 10\% FBS. When cells were $80-90 \%$ confluent, they were passaged at a ratio of 1:3. Exponential growth phase cells from passages 4-8 were used for subsequent experiments.

Groups, treatment and UVB exposure. Cells were either exposed to UVB, exposed to UVB and treated with GL-PS, or non-exposed and untreated. GL-PS $1 \mathrm{mg} / \mathrm{ml}$ was reconstituted in $1 \mathrm{X}$ PBS and was stored at $4^{\circ} \mathrm{C}$. Solutions of different GL-PS concentration were made by diluting in FBS-free DMEM prior to use. All cells were cultured in FBS-free media for $24 \mathrm{~h}$ prior to UVB exposure. Immediately prior to exposure, all media was vacuumed, and cell layers were rinsed 3 times with sterile PBS. Following UVB exposure, matched media was added immediately.

Cell viability assay (MTT assay). Cells were seeded in a 96 -well plate $\left(1 \times 10^{4}\right.$ cells/well), with a volume of $200 \mu 1$ per well. Following attachment to the dish, cells were starved with FBS-free media for $24 \mathrm{~h}$. All media was vacuumed prior to UVB exposure. When exposure was completed, cells were cultured in serum-free media for $24 \mathrm{~h}$, with the specified concentrations of GL-PS where appropriate. MTT was then added $(20 \mu 1,5 \mathrm{mg} / \mathrm{ml})$ and the cells cultured for a further $4 \mathrm{~h}$. Supernatant was then discarded and $150 \mu 1$ dimethyl sulfoxide added. Absorbances were measured at $490 \mathrm{~nm}$ with a microplate reader.

Detection of aging cells using an SA- $\beta$-gal kit. Following UVB exposure and treatment with the specified concentrations of GL-PS, an SA- $\beta$-gal kit was used to stain aging cells according to the manufacturer's instructions. Samples were checked using a light microscope under x200 magnification. For each sample, $>200$ cells from 15 randomly selected fields were checked to calculate the percentage of aging cells (blue stained cell number/total cell number x100).

Measurement of intracellular ROS by flow cytometry. Cells were prepared according to the manufacturer's instructions for use with the ROS detection kit $24 \mathrm{~h}$ after UVB exposure and treatment with GL-PS. Images were captured using a fluorescence microscope, and fluorescence intensity was detected by flow cytometry (FACSCalibur; BD Biosciences, Franklin Lakes, NJ, USA) with BD CellQuest Pro software (version 6.0; BD Biosciences).

Detection of MMP-1 and CICP expression by ELISA. Cell supernatants were collected $24 \mathrm{~h}$ following UVB exposure and treatment with GL-PS, and centrifuged at 1,000 x $g$ for $10 \mathrm{~min}$. ELISA kits were used to detect MMP-1 and CICP concentrations according to the manufacturer's instructions. CICP content was used to represent the Type I collagen content.

Statistical analysis. All experiments were repeated independently at least in triplicate. Experimental data were analyzed with SPSS 19.0 (IBM SPSS, Armonk, NY, USA). Unpaired Students $t$-tests were applied to analyze differences between groups. All data are presented as the mean + standard deviation. $\mathrm{P}<0.05$ was considered to indicate a statistically significant difference.

\section{Results}

Fibroblast viability is reduced dose-dependently by UVB exposure. Fibroblasts were exposed to a range of UVB doses $\left(0,20,40,60,80,120,160\right.$ and $\left.200 \mathrm{~mJ} / \mathrm{cm}^{2}\right)$, then cellular density and changes in condition were observed, and cell 
A

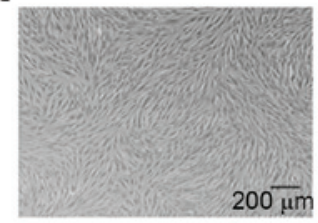

(a) $0 \mathrm{~mJ} / \mathrm{cm}^{2}$

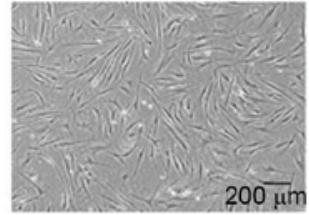

(e) $80 \mathrm{~mJ} / \mathrm{cm}^{2}$

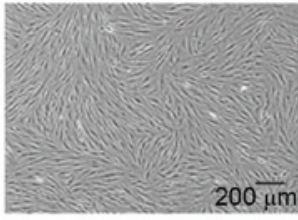

(b) $20 \mathrm{~mJ} / \mathrm{cm}^{2}$

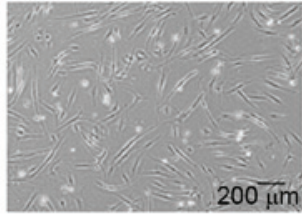

(f) $120 \mathrm{~mJ} / \mathrm{cm}^{2}$

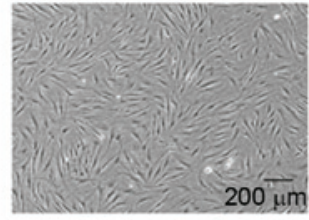

(c) $40 \mathrm{~mJ} / \mathrm{cm}^{2}$

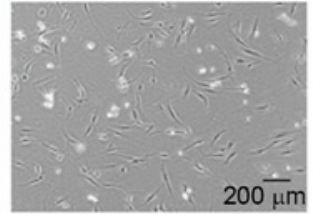

(g) $160 \mathrm{~mJ} / \mathrm{cm}^{2}$

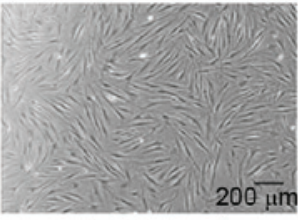

(d) $60 \mathrm{~mJ} / \mathrm{cm}^{2}$

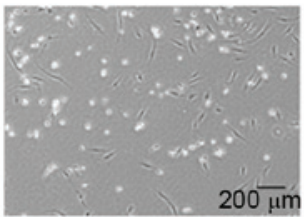

(h) $200 \mathrm{~mJ} / \mathrm{cm}^{2}$

B

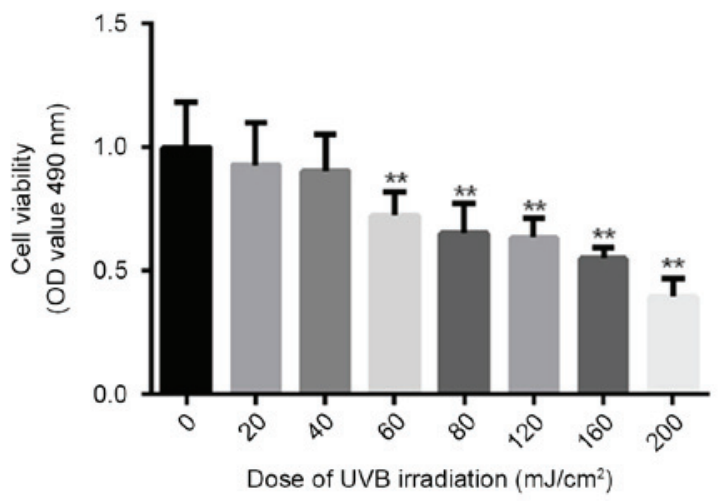

Figure 1. Cell viability of fibroblasts decreases as UVB exposure increases. (A) Cellular density and morphology were observed under a light microscope $48 \mathrm{~h}$ subsequent to UVB exposure. (B) Fibroblast cell viability was measured by MTT assay. ${ }^{* *} \mathrm{P}<0.01$ vs. non-exposed control. Data are presented as the mean + standard deviation. UVB, ultraviolet B.

viability assessed by MTT assay. Following UVB exposure, fibroblast density decreased in a dose-dependent manner (Fig. 1A). In addition, when UVB dosage exceeded $80 \mathrm{~mJ} / \mathrm{cm}^{2}$, a noticeable increase in dead cells was observed (Fig. 1A). A dose-dependent decrease in cellular viability following UVB exposure was also demonstrated (Fig. 1B). The lowest dose to demonstrate significantly reduced cell viability compared with non-exposed cells was $60 \mathrm{~mJ} / \mathrm{cm}^{2}(-27.71 \%$; $\mathrm{P}=0.0083$ Fig. 1B), therefore this dose was used for subsequent experiments.

GL-PS confers protection against UVB-induced cell death to fibroblasts. GL-PS toxicity towards fibroblasts was examined by analysis of cell viability following treatment with different GL-PS concentrations. Compared with untreated cells, increased cell viability was observed in groups treated with 20 , 40 and $80 \mu \mathrm{g} / \mathrm{ml}$ GL-PS $(\mathrm{P}=0.0069, \mathrm{P}=0.0074$ and $\mathrm{P}=0.0037$, respectively; Fig. 2A), but cells treated with $160 \mu \mathrm{g} / \mathrm{ml}$ GL-PS demonstrated no significant difference in viability compared with untreated control (Fig. 2A). Therefore, GL-PS promotes fibroblast viability, but only within a certain range.

Following exposure to $60 \mathrm{~mJ} / \mathrm{cm}^{2} \mathrm{UVB}$, cells treated with 10, 20 and $40 \mu \mathrm{g} / \mathrm{ml}$ GL-PS demonstrated increased viability compared with untreated cells $(\mathrm{P}=0.0025, \mathrm{P}=0.0096$ and $\mathrm{P}=0.0016$, respectively; Fig. $2 \mathrm{~B}$ ), suggesting that GL-PS confers protective effects towards fibroblasts from UVB damage.
GL-PS inhibits UVB-induced fibroblast aging. Aging cells were peri-nuclearly stained blue, using the SA- $\beta$-gal kit (Fig. 3A). The percentage of stained cells was $25.95 \%$ in the UVB non-exposed/GL-PS untreated group compared with $63.53 \%$ in the UVB exposed/GL-PS untreated group $(\mathrm{P}=0.0001$; Fig. 3B). No significant difference in staining was observed in UVB exposed cells treated with $10(57.00 \%)$ and $20 \mu \mathrm{g} / \mathrm{ml}(58.98 \%)$ GL-PS compared with UVB exposed/GL-PS untreated cells (Fig. 3B). However, for UVB exposed cells treated with $40 \mu \mathrm{g} / \mathrm{ml}$ GL-PS, there was a statistically significant decrease in staining compared with UVB exposed/GL-PS untreated cells, with $50.96 \%$ of cells stained $(\mathrm{P}=0.0322$; Fig. 3B).

GL-PS inhibits UVB-induced protein expression of MMP-1 in fibroblasts, and increases protein expression of CICP. MMP-1 protein expression in fibroblasts was significantly increased following UVB exposure compared with non-exposed cells $(\mathrm{P}=0.0097$; Fig. 4A). UVB exposed cells expressed significantly lower levels of MMP-1 protein following treatment with 10, 20 and $40 \mu \mathrm{g} / \mathrm{ml}$ GL-PS compared with untreated cells $(\mathrm{P}=0.0043, \mathrm{P}=0.0007$ and $\mathrm{P}=0.0090$, respectively; Fig. 4A). CICP protein expression levels decreased in fibroblasts following UVB exposure compared with non-exposed cells $(\mathrm{P}=0.0362$ Fig. 4B), but $\mathrm{CICP}$ protein expression levels increased dose-dependently in UVB exposed cells following GL-PS-treatment, with a statistically significant difference 

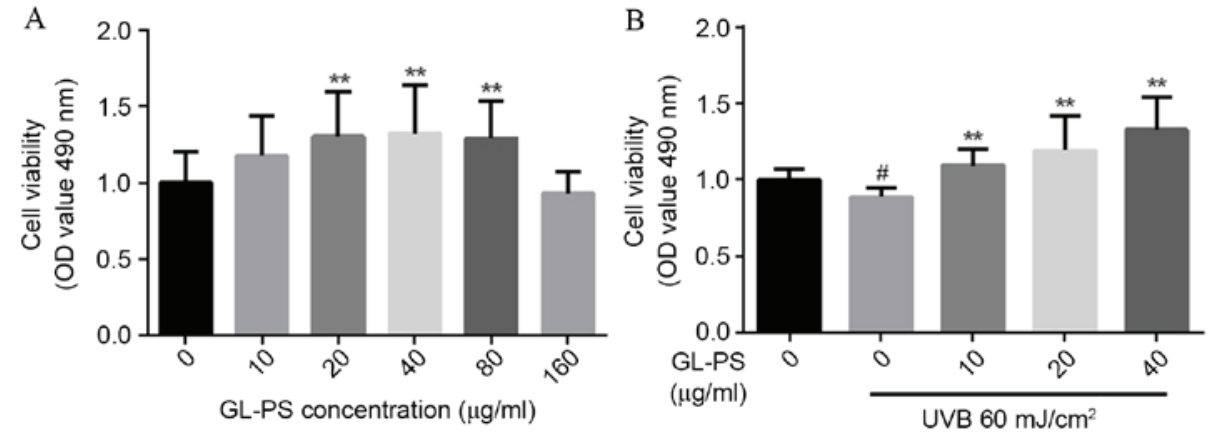

Figure 2. GL-PS protects fibroblasts against UVB-induced cell death. (A) Cell viability following GL-PS treatment was assessed by MTT assay. ${ }^{* *} \mathrm{P}<0.01$ vs. GL-PS untreated control. (B) Cell viability following exposure to $60 \mathrm{~mJ} / \mathrm{cm}^{2} \mathrm{UVB}$, then $24 \mathrm{~h}$ treatment with GL-PS, was assessed by MTT assay. ${ }^{\#} \mathrm{P}<0.05$ vs. UVB non-exposed/GL-PS untreated control. ${ }^{* *} \mathrm{P}<0.01$ vs. GL-PS untreated control. Data are presented as the mean + standard deviation. GL-PS, Ganoderma lucidum polysaccharides; UVB, ultraviolet B.

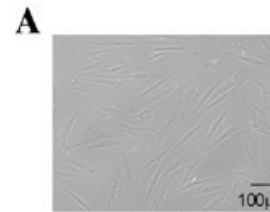

(a) No staining

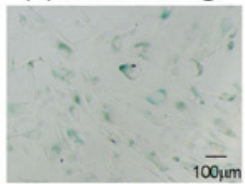

(d)

UVB $60 \mathrm{~mJ} / \mathrm{cm}^{2}$

GL-PS $10 \mu \mathrm{g} / \mathrm{ml}$

\section{B}

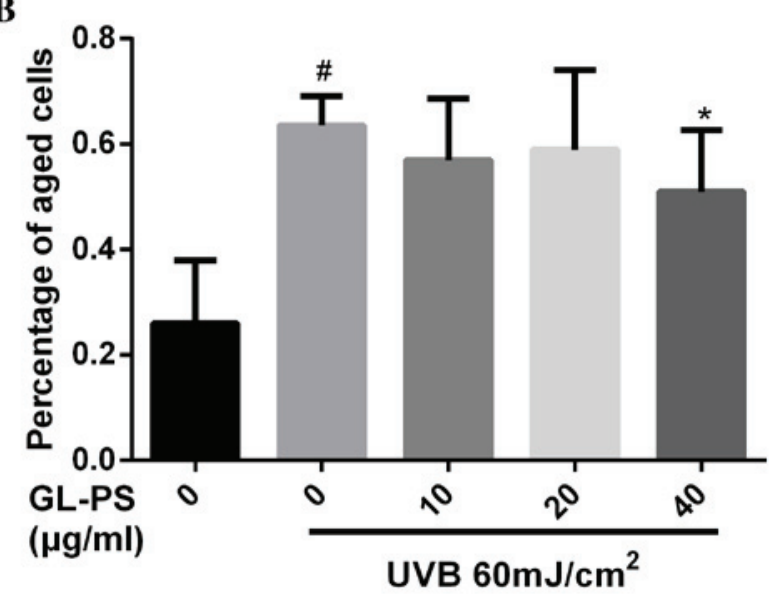

Figure 3. GL-PS inhibits UVB-induced aging in fibroblasts. (A) Representative images of aged cells displaying peri-nuclear blue staining, detected by senescence-associated $\beta$-galactosidase staining. (B) Percentage of aged cells. Data are presented as the mean + standard deviation. ${ }^{\#} \mathrm{P}<0.05$ vs UVB non-exposed/GL-PS untreated cells. ${ }^{*} \mathrm{P}<0.05$ vs. UVB exposed/GL-PS untreated cells. GL-PS, Ganoderma lucidum polysaccharides; UVB, ultraviolet B.

observed in the $40 \mu \mathrm{g} / \mathrm{ml}$ treatment group compared with untreated cells $(\mathrm{P}=0.0074$; Fig. 4B).

GL-PS inhibits UVB-induced ROS in fibroblasts. UVB exposed cells exhibited increased fluorescence compared with non-exposed control cells, indicating increased ROS produc- tion ( $\mathrm{P}=0.0032 ; 2.3$ fold difference; Fig. 5A). Fluorescence decreased in UVB exposed cells as GL-PS concentration increased (Fig. 5A). The flow cytometry results demonstrated a similar trend, with a non-significant decrease in ROS in UVB exposed cells treated with $10 \mu \mathrm{g} / \mathrm{ml}$ GL-PS compared with UVB exposed/GL-PS untreated cells ( $\mathrm{P}=0.313$; Fig. 5B) and significant decreases in UVB exposed cells treated with 20 and $40 \mu \mathrm{g} / \mathrm{ml}$ GL-PS compared with UVB exposed/GL-PS untreated cells $(\mathrm{P}=0.0406$ and $\mathrm{P}=0.0172$, respectively; Fig. 5B). This suggests that above a threshold concentration, GL-PS reduces UVB-induced ROS production.

\section{Discussion}

Ganoderma, a popular traditional drug in Asia, is a member of the Polyporaceae family and is endemic to tropical areas (24). Previous research had revealed multiple pharmaceutical properties of Ganoderma, including antitumor effects (30), immune regulation (31), antioxidative effects (32), hepatoprotective effect against CCl4-induced liver injury (33), and the ability to lower blood sugar (34). A major active ingredient in Ganoderma is GL-PS, which is formed from three single strands of monosaccharide chains, including glucose, galactose, mannose and arabinose, which form a helical three dimensional configuration (35). In traditional Chinese medicine, Ganoderma is used as an anti-aging drug, and modern research has previously demonstrated the anti-aging effects of Ganoderma (26-28). Photoaging refers to accelerated aging of the skin induced by UV light exposure, usually with the involvement of ROS (6). It was therefore hypothesized that the anti-aging effects of GL-PS may derive from inhibition of ROS production; however, to the best of our knowledge, no previous studies had investigated the effects of GL-PS on UVB-induced photoaging. Therefore, in the present study, the protective effect of GL-PS on UVB-induced fibroblast aging was examined.

A UVB toxicity study on fibroblasts revealed that fibroblast cell viability decreased dose-dependently as the UVB dose increased; $60 \mathrm{~mJ} / \mathrm{cm}^{2} \mathrm{UVB}$ was the lowest dose resulting in significantly decreased cell viability, so was selected to create the photoaging fibroblast model. The effect of GL-PS on cell viability was subsequently explored. Treatment of cells that had not been exposed to UVB with 20,40 and $80 \mu \mathrm{g} / \mathrm{ml}$ 

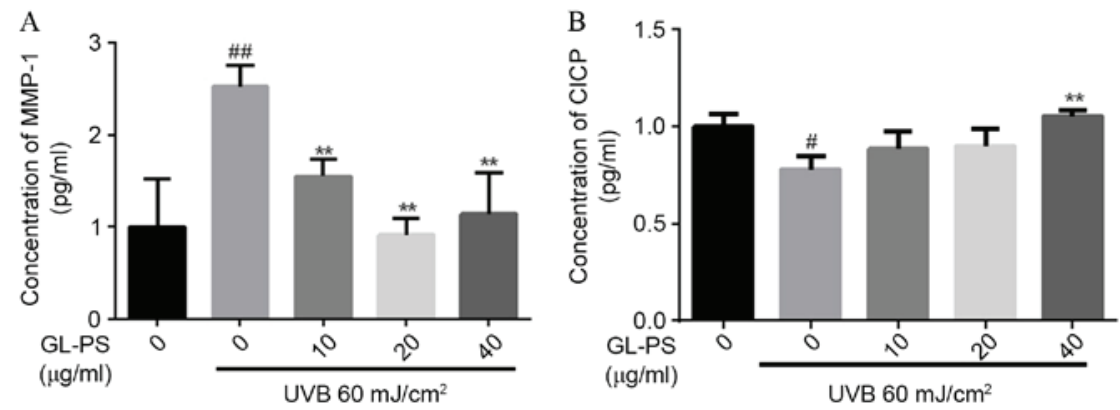

Figure 4. GL-PS inhibits MMP-1 protein expression and increases CICP protein expression in UVB-exposed fibroblasts. (A) MMP-1 and (B) CICP protein expression levels measured by ELISA. Data are presented as the mean + standard deviation. ${ }^{~} \mathrm{P}<0.05,{ }^{\# \#} \mathrm{P}<0.01$ vs. UVB non-exposed/GL-PS untreated cells; ${ }^{* *} \mathrm{P}<0.01$ vs. UVB exposed/GL-PS untreated cells. GL-PS, Ganoderma lucidum polysaccharides; MMP-1, matrix metalloproteinase 1; CICP, C-telopeptide of Type I collagen; UVB, ultraviolet B.

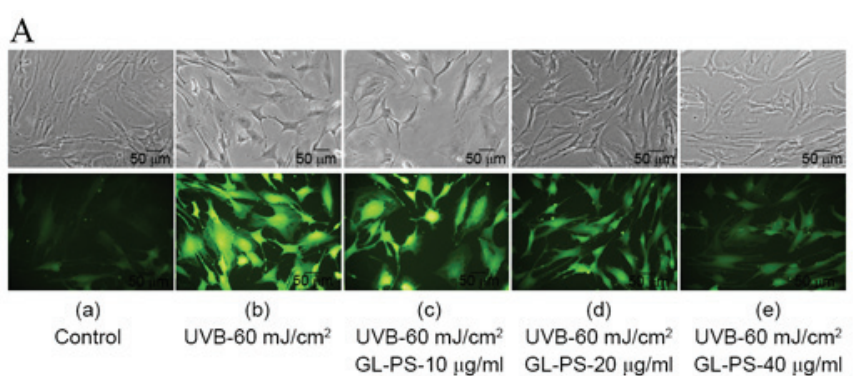

B

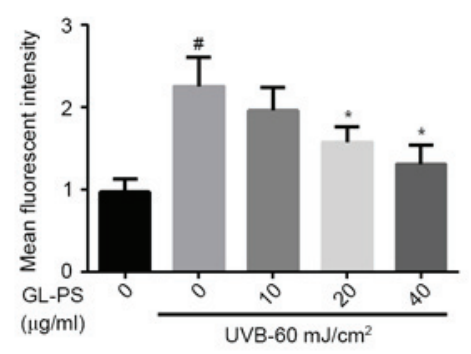

Figure 5. GL-PS inhibits the UVB-induced increase in ROS in fibroblasts. Fluorescence staining and flow cytometry were used to measure ROS levels. (A) Fluorescent staining of ROS-positive cells. (B) Quantitation of ROS levels. Data are presented as the mean + standard deviation. ${ }^{*} \mathrm{P}<0.01$ vs. UVB non-exposed/GL-PS untreated cells. "P<0.05 vs. UVB exposed/GL-PS untreated cells. GL-PS, Ganoderma lucidum polysaccharides; UVB, ultraviolet $\mathrm{B}$; ROS, reactive oxygen species.

GL-PS was demonstrated to promote fibroblast cell viability in excess of untreated cells. Treatment of cells exposed to UVB with 10,20 and $40 \mu \mathrm{g} / \mathrm{ml}$ GL-PS also increased cell viability in excess of UVB exposed cells. GL-PS was reported to prevent apoptosis of pancreatic $\beta$-cells (36). Thus, the ability to inhibit apoptosis of GL-PS may be the possible reason for the increased the cell viability of GL-PS treated fibroblasts. Furthermore, $40 \mu \mathrm{g} / \mathrm{ml}$ GL-PS treatment also significantly decreased the percentage of aged cells, suggesting that GL-PS protects fibroblasts from photoaging.

Photoaging includes epidermal photoaging and dermal photoaging, with dermal photoaging more important to the overall photoaging process (2). Skin wrinkles, induced by decreased extra-cellular matrix components, such as collagens, are a major product of photoaging (37). Collagens are primarily produced by fibroblasts and are a major component of the extra-cellular matrix (16). Previous studies have demonstrated that type I collagen, the most abundant collagen, is degraded by MMP-1 $(14,16,38)$. When degradation of normal collagen and accumulation of abnormal collagen fiber fragments increases, the proliferation of fibroblasts is inhibited, resulting in decreased collagen synthesis $(15,16)$. UVB exposure results in upregulated MMP-1 protein expression and decreased collagen expression (19), which the present study also confirmed. In addition, the present study demonstrated the ability of GL-PS to inhibit UVB-induced MMP-1 protein expression and promote CICP protein expression in fibroblasts. Therefore, GL-PS may be involved in the inhibition of extra-cellular matrix degradation, resulting in protection of skin against photoaging.

Major mechanisms of photoaging include UV-associated DNA damage, and UV-induced increases in cellular ROS. Intracellular chromophores absorb UVB energy and interact with oxygen molecules to produce ROS, resulting in induction of the oxidative stress response and subsequent damage to cells (7-9). Zhao et al (29) demonstrated that GL-PS treatment protects murine skeletal muscles from exhaustive-exercise-induced oxidative stress. In addition, Kao et al (39) isolated low molecular weight $\beta$-1,3-glucan from Ganoderma and demonstrated that this reduces $\mathrm{H}_{2} \mathrm{O}_{2}$-induced intracellular ROS production, and so inhibits inflammation-induced oxidative stress. In the present study, ROS levels were demonstrated to increase significantly in photoaging fibroblasts and, for the first time, GL-PS treatment was demonstrated to inhibit ROS production following UVB treatment.

During the photoaging process, increased ROS levels also activate growth cytokines and their receptors in fibroblasts and keratinocytes, leading to activation of ERK, p38, and JNK in the MAPK pathway $(29,40)$. This promotes MMP expression and decreases collagen levels $(10,11)$. The results of the present study demonstrate the antiphotoaging effect of GL-PS in vitro, however, the effects of GL-PS on the MAPK and other signaling pathways were not investigated. In addition, the efficacy of GL-PS on photoaging in vivo remain to be elucidated. Further studies are required to verify the results of the present study and to assess the underlying mechanism of GL-PS on photoaging.

In conclusion, the present study demonstrates that GL-PS protects fibroblasts from photoaging via its antioxidant ability. This indicates GL-PS treatment may serve as a novel strategy for anti-photoaging. 


\section{Acknowledgements}

The present study was supported by The New Xiangya Talent Projects of the Third Xiangya Hospital of Central South University (grant no. JY201623).

\section{References}

1. Han A, Chien AL and Kang S: Photoaging. Dermatol Clin 32: 291-299, vii, 2014

2. Fisher GJ, Kang S, Varani J, Bata-Csorgo Z, Wan Y, Datta S and Voorhees JJ: Mechanisms of photoaging and chronological skin aging. Arch Dermatol 138: 1462-1470, 2002.

3. Sjerobabski Masnec I and Poduje S: Photoaging. Coll Antropol 32 (Suppl 2): S177-S180, 2008.

4. Hwang E, Park SY, Lee HJ, Lee TY, Sun ZW and Yi TH: Gallic acid regulates skin photoaging in UVB-exposed fibroblast and hairless mice. Phytother Res 28: 1778-1788, 2014.

5. Williams JD, Bermudez Y, Park SL, Stratton SP, Uchida K, Hurst CA and Wondrak GT: Malondialdehyde-derived epitopes in human skin result from acute exposure to solar UV and occur in nonmelanoma skin cancer tissue. J Photochem Photobiol B 132: 56-65, 2014

6. Rittié L and Fisher GJ: UV-light-induced signal cascades and skin aging. Ageing Res Rev 1: 705-720, 2002.

7. Valencia A and Kochevar IE: Nox1-based NADPH oxidase is the major source of UVA-induced reactive oxygen species in human keratinocytes. J Invest Dermatol 128: 214-222, 2008.

8. Emri G, Horkay I and Remenyik E: The role of free radicals in the UV-induced skin damage. Photo-aging. Orv Hetil 147 731-735, 2006 (In Hungarian).

9. Van Laethem A, Nys K, Van Kelst S, Claerhout S, Ichijo H, Vandenheede JR, Garmyn M and Agostinis P: Apoptosis signal regulating kinase-1 connects reactive oxygen species to p38 MAPK-induced mitochondrial apoptosis in UVB-irradiated human keratinocytes. Free Radic Biol Med 41: 1361-1371, 2006.

10. Watanabe H, Shimizu T, Nishihira J, Abe R, Nakayama T, Taniguchi M, Sabe H, Ishibashi T and Shimizu H: Ultraviolet A-induced production of matrix metalloproteinase- 1 is mediated by macrophage migration inhibitory factor (MIF) in human dermal fibroblasts. J Biol Chem 279: 1676-1683, 2004.

11. Quan T, He T, Voorhees JJ and Fisher GJ: Ultraviolet irradiation induces Smad7 via induction of transcription factor AP-1 in human skin fibroblasts. J Biol Chem 280: 8079-8085, 2005.

12. Brennan M, Bhatti H, Nerusu KC, Bhagavathula N, Kang S, Fisher GJ, Varani J and Voorhees JJ: Matrix metalloproteinase-1 is the major collagenolytic enzyme responsible for collagen damage in UV-irradiated human skin. Photochem Photobiol 78: 43-48, 2003.

13. Visse R and Nagase H: Matrix metalloproteinases and tissue inhibitors of metalloproteinases: Structure, function, and biochemistry. Circ Res 92: 827-839, 2003.

14. Gambichler T, Skrygan M, Tomi NS, Breuksch S, Altmeyer P and Kreuter A: Significant downregulation of transforming growth factor-beta signal transducers in human skin following ultraviolet-A1 irradiation. Br J Dermatol 156: 951-956, 2007.

15. Imokawa G: Recent advances in characterizing biological mechanisms underlying UV-induced wrinkles: A pivotal role of fibrobrast-derived elastase. Arch Dermatol Res 300 (Suppl 1): S7-S20, 2008.

16. Quan T, Qin Z, Xia W, Shao Y, Voorhees JJ and Fisher GJ: Matrix-degrading metalloproteinases in photoaging. J Investig Dermatol Symp Proc 14: 20-24, 2009.

17. Afaq $\mathrm{F}$ and Mukhtar $\mathrm{H}$ : Botanical antioxidants in the prevention of photocarcinogenesis and photoaging. Exp Dermatol 15: 678-684, 2006.

18. Katiyar SK: UV-induced immune suppression and photocarcinogenesis: Chemoprevention by dietary botanical agents. Cancer Lett 255: 1-11,2007.

19. Huang J, Luo X, Lu J, Chen J, Zuo C, Xiang Y, Yang S, Tan L, Kang $\mathrm{J}$ and Bi Z: IPL irradiation rejuvenates skin collagen via the bidirectional regulation of MMP- 1 and TGF- $\beta 1$ mediated by MAPKs in fibroblasts. Lasers Med Sci 26: 381-387, 2011.
20. Matsui MS, Hsia A, Miller JD, Hanneman K, Scull H, Cooper KD and Baron E: Non-sunscreen photoprotection: Antioxidants add value to a sunscreen. J Investig Dermatol Symp Proc 14: 56-59, 2009.

21. Sun S, Jiang P, Su W, Xiang Y, Li J, Zeng L and Yang S: Wild chrysanthemum extract prevents UVB radiation-induced acute cell death and photoaging. Cytotechnology 68: 229-240, 2016.

22. Lee KE, Mun S, Pyun HB, Kim MS and Hwang JK: Effects of macelignan isolated from Myristica fragrans (Nutmeg) on expression of matrix metalloproteinase-1 and type I procollagen in UVB-irradiated human skin fibroblasts. Biol Pharm Bull 35: 1669-1675, 2012.

23. Yun TK: Update from Asia. Asian studies on cancer chemoprevention. Ann N Y Acad Sci 889: 157-192, 1999.

24. Sliva D: Cellular and physiological effects of Ganoderma lucidum (Reishi). Mini Rev Med Chem 4: 873-879, 2004.

25. Dudhgaonkar S, Thyagarajan A and Sliva D: Suppression of the inflammatory response by triterpenes isolated from the mushroom Ganoderma lucidum. Int Immunopharmacol 9: 1272-1280, 2009.

26. XiaoPing C, Yan C, ShuiBing L, YouGuo C, JianYun L and LanPing L: Free radical scavenging of Ganoderma lucidum polysaccharides and its effect on antioxidant enzymes and immunity activities in cervical carcinoma rats. Carbohydr Polym 77: 389-393, 2009.

27. Xu Z, Chen X,Zhong Z, Chen L and Wang Y: Ganoderma lucidum polysaccharides: Immunomodulation and potential anti-tumor activities. Am J Chin Med 39: 15-27, 2011.

28. Shi M, Zhang Z and Yang Y: Antioxidant and immunoregulatory activity of Ganoderma lucidum polysaccharide (GLP). Carbohydr Polym 95: 200-206, 2013.

29. Zhao Z, Zheng X and Fang F: Ganoderma lucidum polysaccharides supplementation attenuates exercise-induced oxidative stress in skeletal muscle of mice. Saudi J Biol Sci 21: 119-123, 2014.

30. Yuen JW and Gohel MD: Anticancer effects of Ganoderma lucidum: A review of scientific evidence. Nutr Cancer 53: 11-17, 2005.

31. Lin ZB and Zhang HN: Anti-tumor and immunoregulatory activities of Ganoderma lucidum and its possible mechanisms. Acta Pharmacol Sin 25: 1387-1395, 2004.

32. Yen GC and Wu JY: Antioxidant and radical scavenging properties of extracts from Ganoderma tsugae. Food Chem 65: 375-379, 1999.

33. Kim DH, Shim SB, Kim NJ and Jang IS: Beta-glucuronidase-inhibitory activity and hepatoprotective effect of Ganoderma lucidum. Biol Pharm Bull 22: 162-164, 1999.

34. Seto SW, Lam TY, Tam HL, Au AL, Chan SW, Wu JH, Yu PH, Leung GP, Ngai SM, Yeung JH, et al: Novel hypoglycemic effects of Ganoderma lucidum water-extract in obese/diabetic $(+\mathrm{db} /+\mathrm{db})$ mice. Phytomedicine 16: 426-436, 2009.

35. Nie S, Zhang H,Li W and Xie M: Current development of polysaccharides from Ganoderma: Isolation, structure and bioactivities. Bioactive Carbohydrates and Dietary Fibre 1: 10-20, 2013.

36. Zheng J, Yang B, Yu Y, Chen Q, Huang T and Li D: Ganoderma lucidum polysaccharides exert anti-hyperglycemic effect on streptozotocin-induced diabetic rats through affecting $\beta$-cells. Comb Chem High Throughput Screen 15: 542-550, 2012.

37. Gilchrest BA: Photoaging. J Invest Dermatol 133: E2-E6, 2013.

38. Wang XY and Bi ZG: UVB-irradiated human keratinocytes and interleukin-1alpha indirectly increase MAP kinase/AP-1 activation and MMP-1 production in UVA-irradiated dermal fibroblasts. Chin Med J (Engl) 119: 827-831, 2006.

39. Kao PF, Wang SH, Hung WT, Liao YH, Lin CM and Yang WB: Structural characterization and antioxidative activity of low-molecular-weights beta-1,3-glucan from the residue of extracted Ganoderma lucidum fruiting bodies. J Biomed Biotechnol 2012: 673764, 2012.

40. Chen CC, Chiang AN, Liu HN and Chang YT: EGb-761 prevents ultraviolet B-induced photoaging via inactivation of mitogen-activated protein kinases and proinflammatory cytokine expression. J Dermatol Sci 75: 55-62, 2014. 